\title{
Efficacy and Safety of Different Grafting Types in Endoscopic Sellar Repair
}

Adel R. Al Melesy, ${ }^{1}$ MD.

\author{
* Corresponding Author: \\ Adel R. Al Melesy \\ adelalmelesy@gmail.com
}

Received for publication October 21, 2020; Accepted February 22, 2021; Published online February 22, 2021.

\section{Copyright 2021 The Authors published by Al-Azhar University, Faculty of Medicine, Cairo, Egypt. All rights reserved. This an open-access article distributed under the legal terms, where it is permissible to download and share the work provided it is properly cited. The work cannot be changed in any way or used commercially.}

doi: 10.21608/aimj.2021.47303.1341

${ }^{1}$ Neurosurgery Department, Faculty of Medicine, Al-Azhar University.

\begin{abstract}
Background: Postoperative CSF leakage is one of the most common complications after pituitary adenoma resection by endoscopic endonasal transsphenoidal approach (0.6\%-3.5\%). Sellar reconstruction has been postulated as a crucial step in this approach to overcome such complications.

Aim of the study: to evaluate the efficacy and safety of different grafting types and factors that determine which sellar repair is needed according to the size of arachnoid defect or CSF flow.

Patients and Methods: Retrospective study included 32 patients at $\mathrm{Al}$ azhar university hospitals who underwent endoscopic endonasal sellar repair (EER) with different grafting types after pituitary surgery. 14 patients repair with fascia lata graft, 12 patients with dural substitute (Duragen Integra LifeSciences Corp, USA) and 6 patients vascularized nasoseptal flap.

Results: The mean age was (33.68 \pm 10.2$)$ years, with (59.4\%) females and (40.6\%) males. Regarding post-operative complications; $(21.9 \%)$ of patients had CSF leakage, (6.2\%) had epistaxis and sinusitis, $(9.4 \%)$ had pneumocephalus, and the overall complications rate was (34.4\%), with success rate $(78.1 \%)$, and failure rate $(21.9 \%)$. On comparing the 3 grpups, seven patients $(21.9 \%)$ had repair failure and required reoperation for repair of persistent CSF leak and need recurrent sellar repair, 4 patients (12.5\%) from fascia lata group and 3 patients $(9.3 \%)$ from dural substitute group.

Conclusion: vascularized nasoseptal flap is effective as regards success rate, repair failure and complications rates. The other types (fascia lata and dural substitute) are effective and safe with low CSF leakage rates.

Keywords: Cerebrospinal Fluid Leak; Endoscopic Endonasal; Reconstructions.
\end{abstract}

Disclosure: The authors have no financial interest to declare in relation to the content of this article. The Article Processing Charge was paid for by the authors. Authorship: All authors have a substantial contribution to the article.

\section{INTRODUCTION}

Traditional transsphenoidal surgical treatment is related to a postoperative cerebrospinal fluid (CSF) leak fee starting from zero.5 to $15 \%$. in addition, standard endoscopy-assisted transsphenoidal surgical procedure is associated with a postoperative CSF leak rate of 0.5 to $10 \%$. The expanded endonasal endoscopic approach to the skull base presents the surgeon with excellent visualization and wide access to the cranial base but on the expense of a relatively high rate (5 to 30\%) of postoperative CSF leaks. Despite this potential drawback, the expanded endoscopic endonasal technique has garnered a good deal attention for the resection of midline skull base lesions due to the unparalleled visualization that it offers ${ }^{1}$. The potential to effectively control intraoperative cerebrospinal fluid leak (CSF) is essential to successful transsphenoidal surgery (TSS). In cases without a breach within the diaphragm sellae, the likelihood of developing a postoperative leak is very low and sellar reconstruction is probably useless, because of that, some surgeons prefer to repair patulous arachnoid and correct predictive factors that lead to postoperative CSF leakage ${ }^{2}$. The CSF leakage rate (immediately postoperative leakage) vary widely from 0.1 to $17 \%$, with maximum series reporting rates of $<5 \%$. Patients who increase delayed CSF leaks often undergo repeat surgical procedure or lumbar drainage, and potential complications include meningitis and pneumocephalus. therefore, minimizing those leaks is important now not only to decrease morbidity however to avoid longer hospitalizations for treatment of this complication ${ }^{3}$. Various techniques have been developed to lower the rate of postoperative CSF leaks in surgeries related to intersellar lesions. One technique is the nasoseptal flap, which has been reported to lessen postoperative CSF leaks after pituitary surgeries to $0 \%-2.9 \%$. But, the nasoseptal flap causes some of complications associated with donor site morbidity. The procedure creates a defect of exposed cartilage on the nasal septum. It is difficult to achieve full success, leading to nasal discomfort, anosmia and 
excessive crusting, some techniques try to achieve lower nasal morbidity of the nasoseptal flap by using a free mucosal graft to cover the donor site defect, harvesting mucoperiosteum from either the center turbinate or the posterior septal mucosa and transferring it to the nasoseptal flap donor site ${ }^{4}$.

\section{PATIENTS AND METHODS}

A total of 32 EER patients enrolled in retrospective comparative studyAl-Azhar university hospitals.

Inclusion criterion: Adult patients with pituitary adenoma. Exclusion criteria: Age group younger than 18 years old andSignificant comorbidies (e.g. DM, HTN) . The 32 EER patients were classified according to the used graft into 3 independent groups: Dural substitute group (12 patients),Fascia lata graft group (14 patients) and Vascularized nasoseptal flap group (6 patients)

Patients were subjected to the following: All patients were followed up post-operatively for at least 3 months. The primary outcome was success rate (representing the efficacy of each technique) .The secondary outcomes were: Repair failure rate, Complications rate, such as CSF leakage, infection, and allergy (representing the safety of each technique).

\section{RESULTS}

In the studied population, Regarding basic clinical data; the mean age of all patients was (33.68 \pm 10.2$)$ years. Regarding gender of the patients, the majority $(59.4 \%)$ of patients were females; while $(40.6 \%)$ were males.

Regarding tumor size, (15.6\%) of patients had microadenomas, (40.6\%) had macro-adenomas, and (43.7\%) had giant-adenomas.

Regarding intra-operative data; the mean duration of surgery of all patients was $(165 \pm 23.9) \mathrm{min}$.
Regarding surgical procedures, (37.5\%) of patients had dural substitute, (43.7\%) had fascia lata graft, and (18.8\%) had vascularized nasoseptal flap.

Regarding post-operative complications; (21.9\%) of patients had CSF leakage, (6.2\%) had epistaxis and sinusitis, (9.4\%) had pneumocephalus, and the overall complications rate was (34.4\%) (Figure 1).

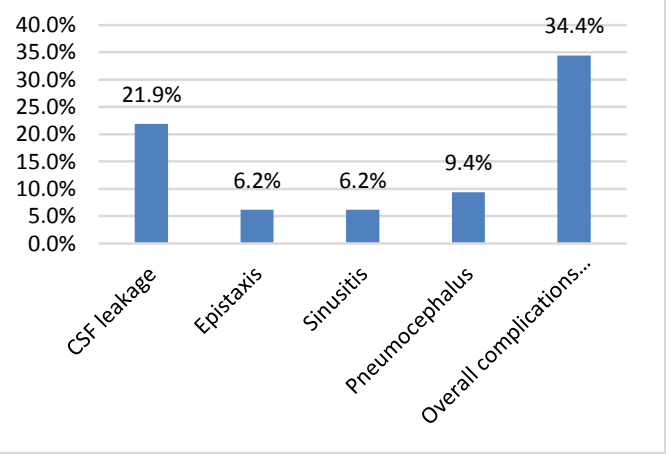

Fig. 1: Post-operative complications among 32 EER patients.

Regarding post-operative outcome data; the success rate was (78.1\%), while the failure rate was (21.9\%). Comparative studies: The 32 EER patients were classified according to the used graft into 3 independent groups:_Dural substitute group (12 patients), Fascia lata graft group (14 patients) and Vascularized flap group (6 patients) .

Regarding basic clinical data; a comparative study between the 3 groups revealed that; A Comparative study between the 3 groups revealed; highly significant increase in duration of surgery in vascularized nasoseptal flap group; compared to other groups ( $\mathrm{p}<0.001)$ (Table 1). Comparative study between the 3 groups revealed non-significant difference as regards age, sex and tumor size ( $p>$ 0.05).

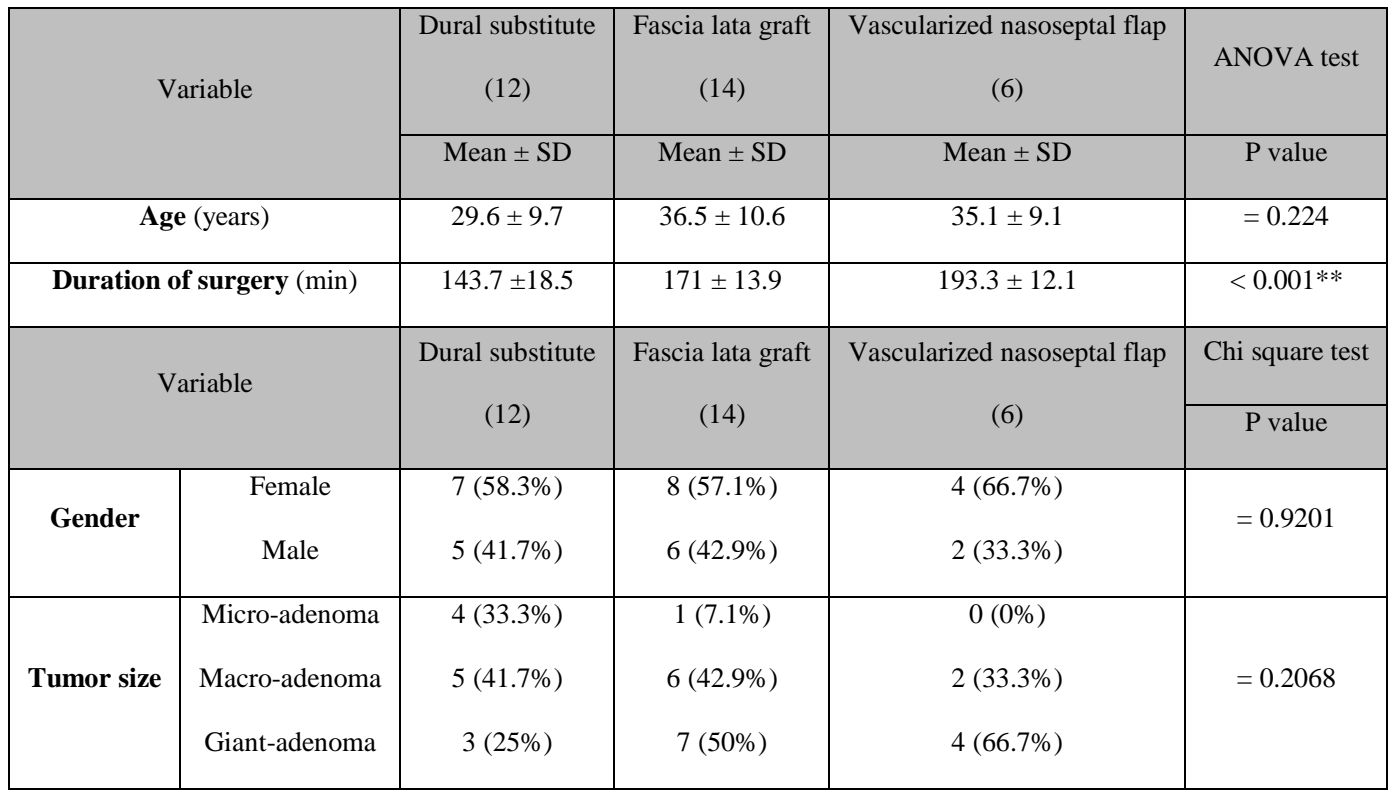

Table 1: Comparison between the 3 groups as regards basic clinical and intra-operative data using ANOVA and Chi square tests . 
Regarding post-operative complications; a comparative study between the 3 groups revealed that;

A Comparative study between the 3 groups revealed; there was increase in complications rate in dural substitute and fascia lata graft groups (50\%, 28.6\% respectively); compared to vascularized nasoseptal flap group (16.7\%),

but without reaching statistical significance ( $\mathrm{p}<0.05$ respectively) (Table 2, Figure 2).

\begin{tabular}{|c|c|c|c|c|c|}
\hline \multicolumn{2}{|l|}{ Variable } & \multirow{2}{*}{$\begin{array}{l}\text { Dural substitute } \\
\qquad(12) \\
3(25 \%)\end{array}$} & \multirow{2}{*}{$\begin{array}{c}\text { Fascia lata graft } \\
\qquad(14) \\
4(28.6 \%)\end{array}$} & \multirow{2}{*}{$\begin{array}{c}\text { Vascularized nasoseptal } \\
\text { flap } \\
(6) \\
0(0 \%)\end{array}$} & \multirow{2}{*}{$\begin{array}{c}\text { Chi square test } \\
\text { P value } \\
=0.3472\end{array}$} \\
\hline CSF leakage & + ve & & & & \\
\hline Epistaxis & + ve & $1(8.3 \%)$ & $0(0 \%)$ & $1(16.7 \%)$ & $=0.3442$ \\
\hline Sinusitis & + ve & $2(16.7 \%)$ & $0(0 \%)$ & $0(0 \%)$ & $=0.1690$ \\
\hline Pneumocephalus & +ve & $1(8.3 \%)$ & $2(14.3 \%)$ & $0(0 \%)$ & $=0.5965$ \\
\hline Overall complications rate & +ve & $6(50 \%)$ & $4(28.6 \%)$ & $1(16.7 \%)$ & $=0.3101$ \\
\hline
\end{tabular}

Table 2: Comparison between the 3 groups as regards post-operative complications using Chi square test:

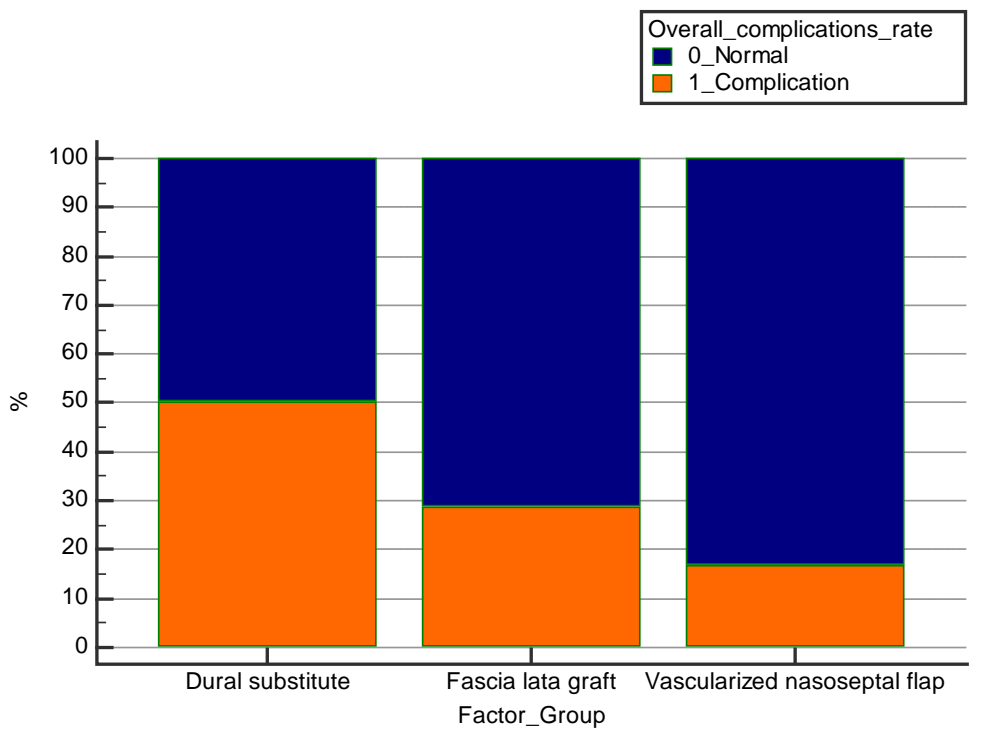

Fig. 2: Comparison between the 3 groups as regards overall complications rate.

\begin{tabular}{|c|c|c|c|c|c|}
\hline \multicolumn{2}{|l|}{ Variable } & \multirow{2}{*}{$\begin{array}{l}\text { Dural substitute } \\
\qquad(12) \\
9(75 \%)\end{array}$} & \multirow{2}{*}{$\begin{array}{c}\text { Fascia lata graft } \\
\qquad(14) \\
10(71.4 \%)\end{array}$} & \multirow{2}{*}{$\begin{array}{l}\text { Vascularized nasoseptal flap } \\
\qquad(6) \\
6(100 \%)\end{array}$} & \multirow{2}{*}{$\begin{array}{l}\text { Chi square test } \\
\text { P value } \\
=0.3472\end{array}$} \\
\hline Success rate (efficacy) & + ve & & & & \\
\hline Failure rate (safety) & + ve & $3(25 \%)$ & $4(28.6 \%)$ & $0(0 \%)$ & $=0.3472$ \\
\hline
\end{tabular}

Table 3: Comparison between the 3 groups as regards post-operative outcome data using Chi square test:

Comparative study between the 3 groups revealed non-significant difference as regards success and failure rates ( $\mathrm{p}$ $>0.05$ ) (Table 3).

Correlation studies to predict efficacy (successful outcome): By using ROC-curve analysis, all 3 graft types showed non-significant predictive values in discrimination of patients with successful outcome from patients without ( $>0.05)$, (Figure 3). 


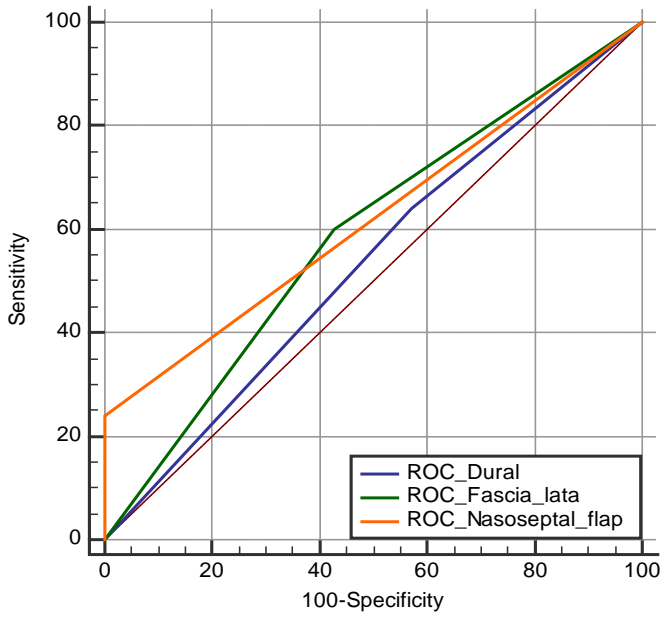

Fig. 3: ROC curve of success rate (efficacy).

Correlation studies to predict safety (un-favorable outcomes): By using ROC-curve analysis, all 3 graft types showed non-significant predictive values in discrimination of patients with complications from patients without ( $\mathrm{p}>0.05$ ), (Figure 4).

By using ROC-curve analysis, all 3 graft types showed non-significant predictive values in discrimination of patients with failure from patients without ( $p>0.05)$, (Figure 5).

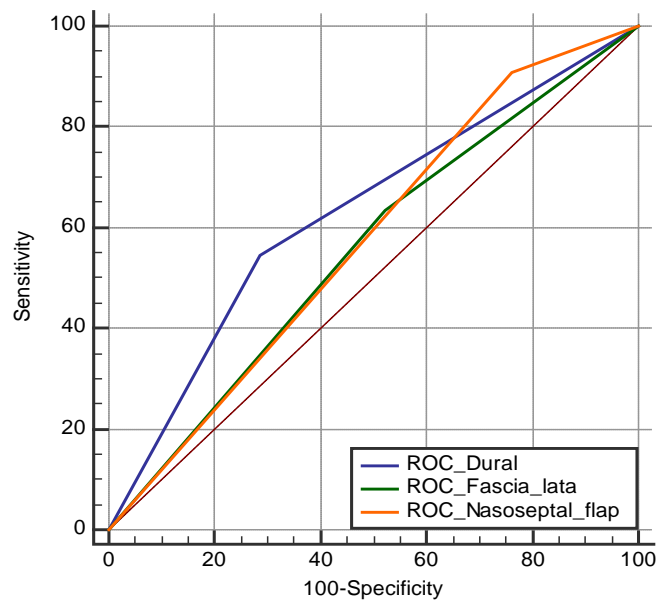

Fig 4: ROC curve of complications rate (safety).

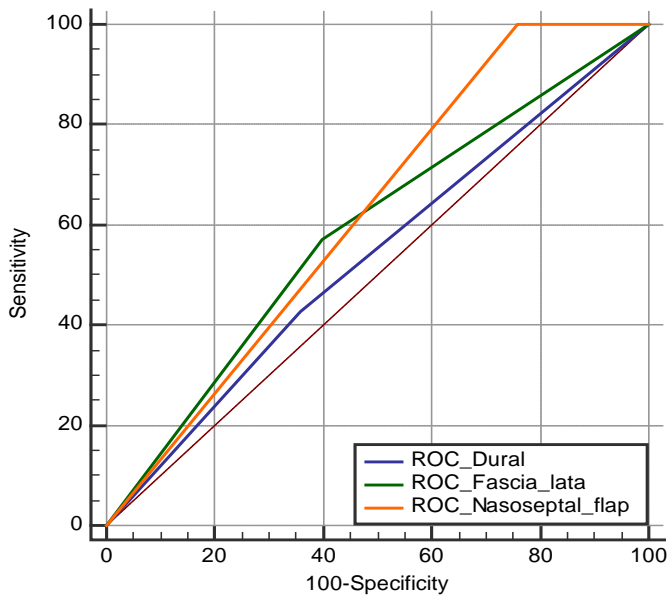

Figure 5: ROC curve of failure rate (safety).

\section{DISCUSSION}

This was a retrospective comparative study conducted on 32 EER patients; to evaluate the efficacy and safety of different grafting types and factors that determine which sellar repair is needed according to the size of arachnoid defect or CSF flow. Regarding basic clinical data; the mean age of all patients was $(33.68 \pm 10.2)$ years. Regarding gender of the patients, the majority $(59.4 \%)$ of patients were females; while (40.6\%) were males. Which came in agreement with Gaynor et al., 2013, Roxbury et al., $2016^{5}$, Scagnelli et al., 2019, Eichberg et al., $2019^{6}$ and Park et al., $2015^{7}$. Gaynor et al., 2013 reported that, a total of 451 cases performed on 429 patients were included in our analysis. The age range of patients was 17 to 85 years (mean age 50.2 years); 173 were male (40.2\%) and 257 (59.8\%) were female. Roxbury et al., 2016 reported that, there were a total of 73 patients who had intraoperative CSF leaks reconstructed with layered vascular grafts. The mean age was 50.7, there were 39 female and 34 male. Scagnelli et al., 2019 reported that, among 158 patients underwent pituitary tumor resection. Median age was 56.5 years, with 70 (44\%) male and 88 (56\%) female patients. Eichberg et al., 2019 reported that, 120 endoscopic endonasal transsphenoidal cases for resection of pituitary adenoma. The average patient age was 53.5 years (range, 20-83). Fifty-four (45\%) patients were male, and 66 (55\%) were female. Park et al., 2015 reported that, between 200 cases in 188 patients (147 pituitary adenoma and 41 other pathologies), 3 cases (1.5\%) were excluded due to preoperative infection. The mean age of the patients was 46.6 years and there were more female patients (54.4\%) . Regarding tumor size, $(15.6 \%)$ of patients had micro-adenomas, (40.6\%) had macro-adenomas, and (43.7\%) had giant-adenomas. Which came in agreement with Roca et al., $2019^{8}$. Roca et al., 2019 reported that, pathological diagnoses and indications for surgery included 275 (72.4\%) pituitary adenomas, 50 Rathke cleft cysts (13.2\%), 12 craniopharyngiomas (3.2\%), 10 arachnoid cysts (2.6\%), 4 chordomas (1.1\%), 3 inflammatory/ autoimmune etiologies $(0.8 \%), 4$ other tumors of the sellar region (1.1\%). Regarding postoperative complications; (21.9\%) of patients had CSF leakage, (6.2\%) had epistaxis and sinusitis, (9.4\%) had pneumocephalus, and the overall complications rate was (34.4\%). Which came in agreement with Munich et al., 2013, Gaynor et al., 2013, Roca et al., 2019 and Park et al., 2015. Munich et al., 2013 reported that, one postoperative CSF leak happened in a patient with a pituitary adenoma, one changed into in a patient with a craniopharyngioma, and one happened in a affected person with a tuberculum sella meningioma .

Gaynor et al., 2013 reported that, CSF leak was observed postoperatively in 18 cases (3.9\%). Of the 291 cases without intraoperative CSF leak, there were 3 (1\%) postoperative leaks. Of those 3 patients, 1 had no repair, 1 had fat graft, and 1 had AlloDerm .

Roca et al., 2019 reported that, CSF leaks are generally controlled via prompt operative exploration of the sellar closure and no longer with lumbar 
drainage. of those 14 sufferers with persistent CSF leaks, one affected person had a single prior transnasal method, with a diagnosis of craniopharyngioma, and one affected person had undergone earlier transnasal operations for an arachnoid cyst

Park et al., 2015 reported that, the commonplace complications after surgical operation had been panhypopituitarism (5.6\%) and diabetes insipidus (3.6\%). There were two meningitis cases without postoperative CSF leaks (1.zero\%) and all patients recovered with an antibiotics therapy.

Comparative study between the 3 groups revealed; there was increase in complications rate in dural substitute and fascia lata graft groups (50\%, 28.6\% respectively); compared to vascularized nasoseptal flap group (16.7\%), but without reaching statistical significance $(\mathrm{p}<0.05$ respectively). Which came in agreement with Gaynor et al., 2013, Munich et al., 2013 and Park et al., 2015.

Gaynor et al., 2013 reported that, they raised the nasoseptal mucosal flap to prevent postoperative CSF leaks. Vascularized mucosal flaps are a very effective, even in high-flow CSF leakage; however, they may bring about increased patient discomfort.25 Anosmia, crusting, and increased process time are deterrents to the usage of mucosal flaps routinely on all transsphenoidal sufferers. In fashionable, these flaps are raised at the start of the method earlier than it's far known if an intraoperative CSF leak will occur, including unnecessary morbidity in cases without a leak. strategies to prepare a flap with minimal septum morbidity were described for cases where a CSF leak isn't expected. Munich et al., 2013 reported that, postoperative CSF leaks occurred in three of 39 primary operations (7.7\%) in which skull base repair turned into performed the use of a mucoperichondrial nasal septal flap. those information recommend that the use of a vascularized mucoperichondrial flap in concert with autologous tissue graft and rigorous interest to acquiring complete coverage of the skull base illness are associated with a decrease rate of CSF leakage following improved endonasal surgical procedure. Park et al., 2015 reported that, the nasoseptal flaprelated complications have been postoperatively reduced olfactory function. VAS scores for olfactory functional disturbance worsened significantly; however, other parameters, including nasal rhinorrhea, sneezing and obstruction, showed no differences after the surgery. Our result came in disagreement with Berker et al., $2013{ }^{9 .}$ Berker et al., 2013 reported that, patients with the dural flap showed no postoperative leaks. But they observed postoperative leakage in $12(10 \%)$ of the 120 patients without the dural flap $(p=0.146)$. They reported that the dural flap reduces the risk of postoperative leak. Comparative study between the 3 groups revealed non-significant difference as regards success and failure rates $(p>0.05)$. Which came in agreement with Scagnelli et al., 2019. Scagnelli et al., 2019 reported that, in patients undergoing free mucosal graft reconstruction, there was a $39 \%$ intraoperative CSF leak rate, with 1 (0.82\%) postoperative CSF leak $(\mathrm{P}<0.05)$. In patients undergoing nasoseptal flap reconstruction, there was a $100 \%$ intraoperative high-flow CSF leak rate, with zero postoperative
Leaks. Our result came in disagreement with Amano et al., $2016{ }^{10}$. Amano et al., 2016 reported that, there are reports on the effectiveness of a nasonasal septal mucosal flap. It is the ultimate method to repair or prevent CSF leakage and enable the extended TSS for the anterior skull base. In general, nasoseptal flap was tougher, thicker, and available to cover larger area than SSM flap. However, affection of normal anatomical structure represents the difference between nasospetal and SSM flaps .

\section{CONCLUSION}

To conclude, vascularized nasoseptal flap is an effective as regards success rate, repair failure and complications rates specially with high-flow CSF leaks with large arachnoid defect. The other two types (fascia lata and dural substitute) is an effective and safe material with low flow CSF leakage rates.

\section{REFERENCES}

1- Munich SA, Fenstermaker RA, et al. Cranial base repair with combined vascularized nasal septal flap and autologous tissue graft following expanded endonasal endoscopic neurosurgery. Journal of Neurological Surgery Part A: Central European Neurosurgery. 2013;74:101-8.

2- Gaynor BG, Benveniste RJ,et al. Acellular dermal allograft for sellar repair after transsphenoidal approach to pituitary adenomas. Journal of Neurological Surgery Part B, Skull Base 2013;74:155.

3- Sanders-Taylor C, Anaizi A,et al. Sellar reconstruction and rates of delayed cerebrospinal fluid leak after endoscopic pituitary surgery. Journal of Neurological Surgery Part B, Skull Base. 2015;76:281.

4- Scagnelli RJ, Patel V, et al. Implementation of free mucosal graft technique for sellar reconstruction after pituitary surgery: outcomes of 158 consecutive patients. World Neurosurgery 2019;122:e506-e11.

5- Roxbury CR, Saavedra T, et al. Layered sellar reconstruction with avascular free grafts: Acceptable alternative to the nasoseptal flap for repair of lowvolume intraoperative cerebrospinal fluid leak. American Journal of Rhinology \& Allergy. 2016;30:367-71.

6- Eichberg DG, Richardson AM, et al. The use of dehydrated amniotic membrane allograft for augmentation of dural repair in transsphenoidal endoscopic endonasal resection of pituitary adenomas. Acta Neurochirurgica. 2019;161:2117-22.

7- Park J-H, Choi JH, Kim Y-I,et al. Modified graded repair of cerebrospinal fluid leaks in endoscopic endonasal transsphenoidal surgery. Journal of Korean Neurosurgical Society. 2015;58:36.

8- Roca E, Penn DL, Safain MG,et al. Abdominal fat graft for sellar reconstruction: retrospective outcomes review and technical note. Operative Neurosurgery. 2019;16:667-74.

9- Berker M, Aghayev K,et al. Management of cerebrospinal fluid leak during endoscopic pituitary surgery. Auris Nasus Larynx. 2013;40:373-8.

10-Amano K, Hori T, Kawamata T,et al. Repair and prevention of cerebrospinal fluid leakage in transsphenoidal surgery: a sphenoid sinus mucosa technique. Neurosurgical Review. 2016;39:123-31. 